\title{
Building Design of Smart Chinese Online Learning Platform Based on Intercultural Communication
}

\author{
Yuan Zhang1*, Zhao Wang² \\ ${ }^{1}$ School of Economics and Management, Xi'an University of Posts \& Telecommunications, Xi'an, China. \\ ${ }^{2}$ School of Chinese Language and LITERATURE, Shaanxi Normal University, Xi'an, China. \\ * Corresponding author. Tel.: +86 18229018999; email: cherryline@163.com \\ Manuscript submitted April 13, 2019; accepted January 27, 2020.
}

doi: 10.17706/ijeeee.2020.10.2.205-213

\begin{abstract}
As the expansion of overseas Chinese learners, distance education of Chinese as a foreign language is getting more and more attention, which gives it a great market potential. Although different kinds of Chinese learning websites are gradually flourishing, there are still plenty of problems need to be solved like education resources, website design and faculty management. In the present paper, we will analyze the characteristics of overseas Chinese learners firstly, and then put forward the building design of smart Chinese online learning platform based on intercultural communication, to provide a new idea for the future development of Chinese distance education.
\end{abstract}

Key words: Teaching Chinese as a foreign language, overseas Chinese learners, online learning platform.

\section{Introduction}

With the overall improvement of China's national economic and cultural strength, Chinese language is playing an increasingly prominent role in international communication, and the market of Teaching Chinese as a Foreign Language (TCFL) is also expanding year by year. At present, TCFL education can be roughly divided into two forms: first is the classroom teaching, including Chinese learning classes in various universities, private Chinese training institutions and overseas Confucius institutes; second is the remote education relying on modern information technology. Due to rich resources, remote teaching and easy-to-use, Chinese learning websites have become an important form of remote Chinese education in recent years. It should be noted that, TCFL education is still based on traditional classroom teaching and mainly serves foreigners who are studying, living or working in China, while remote teaching mostly plays an auxiliary role. Most of Chinese learning websites only transfer the classroom teaching content to the network platform without fully considering the characteristics and needs of overseas Chinese learners, and also need to be improved in the website resources, faculty resources, registration flows and so on [1].

According to above problems, this paper will take overseas Chinese learners as main object, and propose suggestions for building the smart Chinese online learning platform which based on intercultural communication.

\section{Main Characteristics of Overseas Chinese Learners}

Up to 2017, 146 countries and regions have set up 525 Confucius institutes and 1113 Confucius classrooms [2]. At the same time, the number of primary and secondary schools offering Chinese language courses is also increasing sharply, which is eight times that of higher education institutions [3]. Overseas 
Chinese learners have become a group in such vast numbers that cannot be ignored. Compared with Chinese language learners in China, overseas Chinese learners have their own characteristics.

\subsection{Different Language and Cultural Environment}

Overseas Chinese learners are surrounded by their native language and culture environment. They can only indirectly contact the Chinese social and cultural information from public media or other ways beyond Chinese language classes, it is easy for them to form cultural prejudice or misunderstanding of Chinese culture and Chinese language due to media orientation and personal thinking habits.

\subsection{Fragmented Learning Time}

Generally, overseas Chinese learners spend less time on studying. Students can hardly communicate in Chinese outside the Chinese class per week, which makes their Chinese input quite limited. For example, the Chinese classroom teaching time of 6 primary schools with Chinese course in Chiengmai (Thailand) is only 1-3 hours per week, and less than $8 \%$ of students would take more time for studying Chinese outside of classroom [4]. It could be worse for those people who have left campus and gotten a formal job, they are difficult to have enough time and energy to participate in systematic Chinese language courses.

\subsection{Interest Is the Main Motivation for Learning}

Overseas Chinese learners are composed of students with Chinese origin and students with non-Chinese origin. Students from Chinese immigrant families usually learn Chinese because of their parents' requirements or work needs [3]. While for non-Chinese peers, more people just learn Chinese out of interest or curiosity in addition to those people who will come to China for short-term exchanging, business activities or translation work in the future. In the reports for the learning motivation of overseas Chinese learners, interests of Chinese language and culture is the second most important type after career development or future opportunities [5]-[7]. Therefore, non-Chinese peers usually have strong learning initiative in the short term [8]. Once they encounter difficulties in learning process, it has a great possibility that they will directly choose to give up.

\subsection{Fewer Study Partners}

Most Chinese language learners from Chinese immigrant families could get learning partners from their families or Chinese communities. Although most of non-Chinese peers have a great desire to have fixed learning partners, they have less chance to find it outside of classroom because of the limitation of social network and community culture [8], [9], which might affect their motivation and persistence for learning Chinese.

\section{Necessity and Feasibility Analysis of Building Smart Chinese Online Learning Platform}

\subsection{Necessity of Building Smart Chinese Online Leaning Paltform}

In accordance to the previous discussion, it has large potential overseas Chinese learning population as the increasing of China's national and economic strength. But current Chinese teaching and learning methods could not fully satisfy the learning needs and characteristics of overseas Chinese learners, which mainly manifest in the following three aspects.

\subsection{Time, Teacher Resources and Space Constrains}

Although the number of overseas Confucius institutes and educational institutions offering Chinese courses is increasing year by year, class capacity and teacher resources are still quite limited. In the traditional classroom teaching, the location of educational institution is fixed, each classroom has only 
20-30 students, and a teacher could only teach one class at a time. At the same time, learners need to set aside blocks of time and money to join in Chinese classes. Both of them greatly increase the time and economic cost of learning Chinese, and make a lot of people to give up the final goal of mastering Chinese. The similar situation is also exists in Chinese learning. At present, most of Chinese learning websites are just copy the design of concrete course system and curriculum of Chinese classroom teaching, which does not utilize students learning time fully and effectively even though it has solved the space and teacher resources constrains.

\subsubsection{Lack of immediate feedback}

This is the main disadvantage of current Chinese online courses. Most of Chinese learning websites or open course resources online could provide teaching videos and digital textbooks, while only few websites have online face-to-face teaching, Q\&A and feedback functions, which abandoned the greatest advantage of traditional classroom teaching [10]. To solve this problem, it needs to expand scale of teaching resources, update existing database on time, and further supplement professional faculty.

\subsubsection{No unified standard for grading teaching resources}

Up to now, there are plenty kinds of Chinese language textbooks and learning materials compiled by different educational institutions for oversea learners on the market. Most of them are based on the examination outline of Hanyu Shuiping Kaoshi (HSK), while grading standards and course design are not the same. Take Thailand as example. Three kinds of Chinese textbooks are used in the 6 primary schools with Chinese course in Chiengmai, they were compiled separately by Thai native educators, Chinese native educators and cooperated by educators from these two countries [4]. Each of them has different content, key points and course arrangement, and leads to different learning outcomes. Same situation also consists in the online learning websites. A number of websites include learning materials without classification, grading and inspection, which makes information resources confusion and full of errors, and bring negative influences for Chinese overseas learners.

Therefore, it is necessary to change the traditional course-oriented online education mode, to design personalized Chinese courses based on their own interest points and learning needs under the perspective of intercultural communication. At the same time, the new learning platform also needs to establish a good learning effect evaluation \& feedback system and online learning community to help learners build their confidence and consistent learning motivation.

\subsection{Feasibility of Building Smart Chinese Online Leaning Paltform}

In terms of feasibility, both of existing digital technology and TCFL faculty resources can guarantee the design and operation of Chinese online learning platform.

Firstly, at the technical level, there are numerous techniques available. For example, current digital resource processing technology could transform massive written Chinese textbooks, audio, video or other kinds of learning resources into digital, standardized and fragmented digital education resources, which could make them composable and reusable; Digital media convergence technology could easily integrate HTML web pages, servers, blogs, PDFs and smartphone applications into only one page, to make it more convenient for learners to search, copy and integrate required Chinese learning resources; Cloud server and cloud computing collaborative operation can not only solve the problem of complicated coordination of content, security and operation of Chinese teaching materials, but also effectively connect multiple Chinese learners to realize multi-person sharing of learning materials and build online learning communities; Smart mobile devices and application development technology also provide safeguard for the instant transmission of educational information.

At the teacher resource level, 19 Chinese international promotion bases have been established in China, 
and 63 colleges and universities have set up post-graduate program of Chinese International Education [11]. It also should be noted that, China National Office for Teaching Chinese of Foreign Language (NOCFL) promulgated the standards of international Chinese language teacher in 2007 and 2014 [12], and restarted examination of TCSOL. These measures make the construction of faculty for international Chinese education further standardized and specialized. Meanwhile, thousands of Chinese overseas students also could be potential spreaders of Chinese language and culture. They will become natural tutors and learning partners for overseas Chinese learners after received some training.

\section{Functional Requirements of Smart Chinese Online Learning Platform}

Smart Chinese online learning platform is an e-education product to provide overseas Chinese language education services, which relies on modern digital technology and mobile devices such as personal computers, tablet computers and smart phones. Through, the platform could provide online teaching, testing, self-adaptive learning and reviewing, and automatically analyze the learning obstacles and problems of Chinese learners on the basis of real-time evaluation of teaching results, then provide personalized learning programs for learners.

Consequently, this platform should contain following functions:

\subsection{Resource Integration}

The platform shall integrate various types of Chinese international education resources. These resources should be stored and managed according to themes or types of curriculum, and updated on time.

\subsection{Teaching Management}

The system of platform will organize and arrange curriculum, learning tests and learning guidance according to learners' needs. It will also regularly push relevant learning materials like news reports, popular science articles and videos based on learners' interests.

\subsection{Evaluation and Feedback}

Learning objective system will be set for all courses in the platform. After learning the content of each unit, learners need to complete the exercise test. Evaluation system will record and analyze testing results, then feed back to learners whether they have reached the learning goal, what problems they might have in learning and what learning materials could be used to solve these problems.

\subsection{Interactive Discussion}

This platform could provide online or offline interactive discussion services between different learners or between learners and teachers, including online sharing of learning materials, creating learning groups, offline face-to-face communication counseling and so on.

\section{Design and Construction of Smart Chinese Online Learning Platform}

\subsection{Modular Design of the Platform}

This platform will include four different systems based on the functions listed above.

\subsubsection{Chinese digital education resource database}

This module will be constituted by digital resource database and cloud server, and it could be treated as the foundation and core of the whole network platform. Different kinds of authorized education materials, such as written textbooks, audio files, classroom teaching recording and related HTML web pages, will be stored in the resource database after digital processing. By using the cloud server of database, all of digital resources in this platform not only could be updated and statistical processed regularly, but also be 
deployed and integrated easily for curriculum design.

\subsubsection{Education management system}

This module is used for management and individualization design of curriculum. After completing the platform registration, learners will fill in the Chinese learning intention questionnaire online. According to their interests, learning objectives and daily schedule, this system will allocate relevant teaching resources from Chinese digital education resource database to design and provide available curriculum plans. Meanwhile, the learning objective system of the curriculum will be established according to the requirements of curriculum standards. Education management system will consist of knowledge guidance module, exercise module, evaluation module and learning management module. For knowledge guidance module, it will allocate and organize all kinds of teaching materials for learners based on the learning units of curriculum they participate in. When learners are found to have not reached their learning goals, it could provide relevant learning materials to achieve their goals. For exercise module, it will mark the learning objective for every exercise under the learning objective system, to ensure that the distance between learners' learning results and learning objectives could be found through tests, and provide practice plans for different learners to help them achieve their learning goals at the same time. For evaluation module, it will accurately evaluate learners' learning results by post-tests or progress tests. For learning management module, it will prepare and deploy learning resources and tests from the knowledge guidance module and exercise module respectively according to learning objectives and curriculum standards. After learners completed post-tests, it will command evaluation module to make real-time evaluation, and mobilize the knowledge guidance module and exercise module to prepare personalized learning plans for learners, thus complete the Chinese mart learning process.

\subsubsection{Faculty management system}

It is used for the management and deployment of Chinese teachers in the platform, including three parts: the general faculty database, assessment module and teaching management module. The general faculty database will store and manage the basic information of all TCFL teachers in the platform. The assessment module will be responsible for evaluating teachers' professional fields and teaching ability, and tracking learners' post-tests results and evaluation about teachers' tutoring regularly, so as to assess teachers' guidance or counseling to students. Professional fields include listening, speaking, reading, writing and culture. Teaching ability will be divided into five levels: zero foundation, elementary level, intermediate level and advanced level. All TCFL teachers who agree to enter the platform shall pass the test of the assessment module and complete the teaching ability classification firstly. The assessment module will regularly integrate learners' evaluation of teachers and decide whether need to improve or lower the teaching ability of teachers. Teaching management module will be responsible for teacher deployment, curriculum management, teacher-student interaction, and reward and punishment settlement. When learner requests manual guidance from teacher, the teacher management module will search for faculty who can provide online guidance or who is in the vicinity of this learner to provide face-to-face instruction. Teacher will get paid when tutoring completed. The time of duration, content and evaluation of tutoring this time will be recorded as the main basis for regular assessment of teacher. Teachers can also review the course they have tutored, search the tutoring tasks they could get and all the comments from learners through this module.

\subsubsection{Learner system}

It is in charge for learners' self-course management and multi-person interaction, including individual center, course learning module and learning group module. Through the personal center, learners can manage their basic information, choose available courses and join in study groups. The course learning module is responsible for receiving course resources, post-tests and daily learning materials allocated by 
the education management system, then feeding evaluation about learners' learning results back to it. The learning group module could help learners search for learning partners in the platform, establish learning groups, and complete online discussion or resource sharing.

\subsection{Structural Design of the Platform}

Complete structure of Smart Chinese online learning platform is shown as Figure 1. This platform can be downloaded as a software program and installed on a PC, or installed as an application on a tablet or mobile smart phone. Learners can access platform through mobile Internet to complete distance learning, download learning resources, conduct post-tests and interact with learning partners or teachers.

The Chinese digital education resource database is responsible for providing learners with a large number of learning resources marked with learning objectives, which is the main way to make profits. Teachers will receive learners' online or offline guidance requests through faculty management system, they can also download teaching resources directly from the resource database, or upload learners' learning problems or suggestions about curriculum for analysis if necessary.

Learners can also download the learning resources from the resource database, or upload their learning results and obtain the learning resources after analyzed by it.

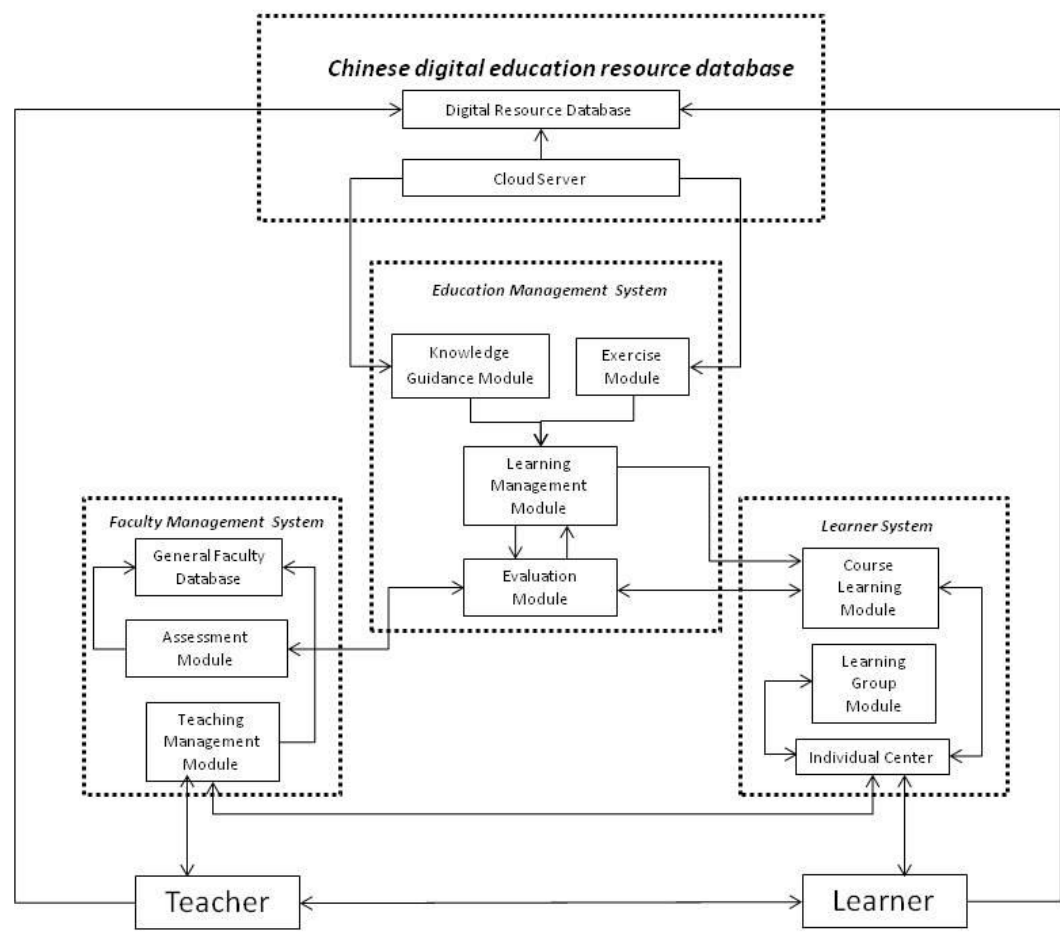

Fig. 1. Structural design of smart Chinese online learning platform.

\section{Analysis of Core Issues}

This learning platform will integrate teaching, practice, evaluation and interactive discussion, to help learners acquire resources on demand, obtain evaluation of learning effect in time, and construct interpersonal network. Although current digital technology and modern education technology are enough to support the design and operation of this platform, there are still many problems, mainly including following three aspects.

\subsection{Formulating Curriculum Standards and Learning Objectives}


Although there are a great variety of guidance materials for Chinese international education on the market, most of them are self-compiled by colleges and universities based on the HSK syllabus. The content arrangement is not completely consistent, and interpretations for some knowledge dots are different too. Therefore, it is necessary to establish a systematic classification standard when classifying and sorting out the educational materials in the Chinese digital education resource database. To achieve the unity of courses' syllabus and content, we should grade and classify Chinese learning resources reasonably based on the examination outline of HSK, trying to find appropriate explanations for the controversial knowledge dots. These tasks will be very detailed and tedious, which require integration of existing research finds for Chinese language and TCFL and complicated cooperation of operators, technicians, publishing companies and research institutions of universities, but they are the foundation stone for this platform and must be completed. More specifically, the first thing we should do is recruiting TCFL teachers and Chinese language researchers to formulate Chinese syllabus and unify concrete teaching content, and then negotiating textbook copyright with publishers. Finally, we need to rely on technicians to digitize and organize these learning resources in the database.

\subsection{Evaluating the Teaching Ability of Chinese Language Teachers Accurately}

Although the NOCFL has proposed the standards for international Chinese language teachers, the curricula of international Chinese language education in colleges and universities are quite different, which leads to individual variance for international Chinese teachers about their expertise, teaching experience and teaching ability. Hence, the accurate evaluation of teaching ability is the key link during recruiting teachers. In this regard, it is necessary to set up a review team which consists of teachers with rich experiences in the field of TCFL. They should strictly comply with the requirements of the standards made by NOCFL, and timely eliminate unqualified candidates according to the scoring results in the evaluation module, so as to ensure that teachers can give full play to their capabilities in their most professional fields.

\subsection{Self-adaption and Self-organizing of Learning Management Module}

Learning management module is the key point in the education management system. It's in charge of setting optimal and feasible curriculum plans according to learners' interest, knowledge base and learning motivation, allocating appropriate learning resources through knowledge guidance module and exercise module, and formulating corresponding solutions for learns' learning difficulties based on the assessment of evaluation module. Therefore, the main issues here are to ensure that the learning management module could accumulate the existing operating experience, and has the ability of self-organizing to adjust its own structure and behavior parameters in the face of changes in the external environment, and coordinate the other three modules by itself. The technology used in this module might be partly referred to automatic recommendation function of Taobao, which changes the tests' results and interests of learners into keywords, search related learning resources and design personalized learning program automatically.

\section{Epilogue}

With the rapid development of Internet technology, Chinese online education has solved the problem of remote Chinese teaching. As the coming of $5 \mathrm{G}$ era, remote point-to-point digital services will be truly realized, and will bring Chinese online education more changes on educational technologies. Through conceptual innovation and technological reformation, online learning platform will be able to put learners' actual needs first. By means of tracking learners' learning outcomes on real-time, and creating personalized learning plans, it will realize the ultimate goal of "teaching students in accordance of their aptitude", which has been highly praised by many educators in the history. In the meantime, more efficient and scientific Chinese language education could further share China's cultural wealth and future opportunities to the 
whole world, and bring huge social and economic benefits in the future.

\section{Conflict of Interest}

The authors declare no conflict of interest.

\section{Author Contributions}

Yuan Zhang conducted the research and Zhao Wang analyzed the data. We wrote the paper together. All authors had approved the final version.

\section{References}

[1] Luo, S., \& Cai, H. (2013). Research on educational resource module design problem of network in teaching Chinese as a foreign language. Overseas Chinese Education, 3(68), 290-295.

[2] Lu, D. (2018). On structuring and expanding the meaning of confucius institute. Studies on Cultural Soft Power, 3(5), 40-48.

[3] Li, Y. (2018). Some thoughts on the younger-age trend of overseas Chinese learners. Chinese Teaching in the World, 3(32), 291-301.

[4] Wang, D. (2018). Investigation Report on Chinese Teaching in Primary Schools in Chiang Mai, Thailand. Undergraduate dissertation, Tianjing Normal University, Tianjing.

[5] Ding, A. (2015). Analysis on the change of motivation of Chinese learners with enhanced motivation in the target language environment. Applied Linguistics, 2, 116-124.

[6] Kim, H. (2018). Survey on Chinese Teaching in Tunisia. Unpublished undergraduate dissertation, Dalian University of Foreign Languages, Dalian.

[7] Yoanna, A. M. (2018). The Study on Spoken Chinese Learning Strategies of Indonesian Students in Non-Chinese language Environment. Undergraduate dissertation, Shanghai International Studies University, Shanghai.

[8] Zhang, J. (2018). A contrastive analysis of learning needs between ethnic Chinese learners and non-ethnic Chinese learners. Journal of Yunnan Normal University (Teaching and Research on Chinese As A Foreign Language), 16(6), 22-29.

[9] Sun, Y. J. (2018). A Comparative Study of Chinese Teaching in Thailand - A Case Study of Chinese Programs in Pathumthep School. Unpublished undergraduate dissertation, Zhengzhou University, Zhengzhou.

[10] Li, G. (2017). Analysis and Designing of Network Teaching Website for Teaching Chinese as a Foreign Language. Unpublished undergraduate dissertation, Yantai University, Yantai.

[11] China National Office for Teaching Chinese of Foreign Language. National Base for the International Promotion of Chinese Language. Retrieved from the website: http://english.hanban.org/node_9842.htm

[12] China National Office for Teaching Chinese of Foreign Language. International Standards for Chinese Language Teachers. Retrieved from the website: http://english.hanban.org/node_9906.htm.

Copyright (C) 2020 by the authors. This is an open access article distributed under the Creative Commons Attribution License which permits unrestricted use, distribution, and reproduction in any medium, provided the original work is properly cited (CC BY 4.0). 
Yuan Zhang was born on Decmber 3, 1988 in Xi'an. She was finishing her master program in City University of Hong Kong in December of 2012. Her major includes marketing and electronic commerce.

She has worked as lecturer in School of Economics and Management, Xi'an University of Posts \& Telecommunications in China since July of 2013. Lastest publication includes: Yuan Zhang, Yangzi Fan, Zhang Quan, Empircal research of "four modernizations" coordination level in Shaanxi province based on the theory of the coupling, Journal of Xi'an University of Posts and Telecommunications, Sep, 2015. Her current research interests include e-commerce, consumer behavior and industrial economy.

Ms. Zhang is a member of both Chinese Marketing Association and Shaanxi Economic Association. She got second prize of Shaanxi Provincial Science and Technology Award on February $4^{\text {th }}$ of 2017.

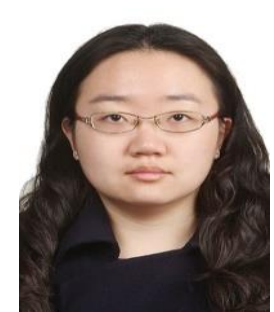

Zhao Wang was born on July, 1989 in Xi'an. She was finishing her PhD program in the Chinese Language and Culture College of Beijing Normal University in January of 2019. Her major includes teaching Chinese as a foreign language.

She has worked in School of Chinese Language and Literature, Shaanxi Normal University in China since June of 2019. Lastest publication includes: Zhao Wang, Xin Yan, Yanni Liu, Gregory J. Spray, Yuan Deng, Fan Cao, Structural and functional abnormality of putamen in children with developmental dyslexia, Neuropsychologia, 2018, in press. Fan Cao, Xin Yan, Zhao Wang, Yanni Liu, Jin Wang, Gregory J. Spray, Yuan Deng. Neural signatures of phonological deficits in developmental dyslexia[J]. Neuroimage, 2017, 146:301-311. Her current research interests include linguistics, language cognitive, teaching Chinese as a foreign language, Chinese developmental dyslexia. 